\title{
«Tentato storicismo»: Enzo Siciliano lettore indocile di Bassani
}

"Tentato storicismo": Enzo Siciliano Uncomfortable Reader of Bassani

"Tentato storicismo » : Enzo Siciliano lecteur indocile de Bassani

Valter Leonardo Puccetti

\section{OpenEdition}

\section{Journals}

Edizione digitale

URL: http://journals.openedition.org/cei/3866

DOI: $10.4000 /$ cei.3866

ISSN: 2260-779X

Editore

UGA Éditions/Université Grenoble Alpes

Edizione cartacea

ISBN: 978-2-37747-040-2

ISSN: 1770-9571

Notizia bibliografica digitale

Valter Leonardo Puccetti, ««Tentato storicismo»: Enzo Siciliano lettore indocile di Bassani», Cahiers d'études italiennes [Online], 26 | 2018, online dal 28 février 2018, consultato il 26 mars 2021. URL: http://journals.openedition.org/cei/3866 ; DOI: https://doi.org/10.4000/cei.3866

Questo documento è stato generato automaticamente il 26 mars 2021

(c) ELLUG 


\title{
«Tentato storicismo»: Enzo Siciliano lettore indocile di Bassani
}

\author{
"Tentato storicismo": Enzo Siciliano Uncomfortable Reader of Bassani \\ «Tentato storicismo » : Enzo Siciliano lecteur indocile de Bassani
}

Valter Leonardo Puccetti

1 Niccolò Gallo presentò Enzo Siciliano, appena laureato in filosofia e fattosi conoscere per una recensione delle Ceneri di Gramsci di Pasolini, a Giorgio Bassani ${ }^{1}$ che lo introdusse nella rivista «Paragone» (dove Siciliano pubblicò il racconto Un'avventura mancata, poi estromesso dalla sua opera prima in volume in cui figura invece Il ricatto, uscito sulla stessa rivista), ne mediò l'amicizia coi Bertolucci, Attilio e il figlio Bernardo $^{2}$, ne pubblicò la traduzione di Tarr di Wyndham Lewis per Feltrinelli, di cui Bassani era direttore editoriale defacto, ne lesse i primi racconti che apprezzò ma sottopose, prima di pubblicarli nel volume Racconti ambigui, a un crivello estenuante di correzioni, come era solito fare. Ecco il ricordo di Antonio Debenedetti:

Una volta, incrociando Enzo Siciliano che usciva da un incontro con Giorgio, intento a rivedere i Racconti ambigui che avrebbe poi pubblicato in una collana da lui diretta, lo trovai con le occhiaie che gli arrivavano al mento, stanchissimo, quasi incapace di parlare. Che cosa fosse successo posso solo ricostruirlo e immaginarlo sulla base di esperienze personali. Bassani editor poteva a volte, decidendolo all'improvviso, mettersi a leggere con voce piena e forte un tuo capoverso facendo delle sottolineature con la sua dizione. Quella lettura diventava all'improvviso uno specchio nel quale vedevi riflessi, ingigantiti e finalmente lampanti certi tuoi difetti naturali [...] Ti rendevi conto di quelli che difetti non erano ma anzi costituivano delle tue prerogative da non disprezzare anche se potevano inquietare per la loro originalità. Saltavano purtroppo fuori anche le goffaggini, peggio i malvezzi nati dal poco rispetto di sé o da una sottovalutazione del proprio lavoro di scrittore ${ }^{3}$.

2 La pedagogia editoriale di Bassani, ricorda ancora Debenedetti, era allusiva e per così dire gestaltica, non puntuale, non 'illuministica', e mirava a una rivisitazione contestuale dell"errore':

Bassani finiva così con l'insegnare moltissimo ma a un prezzo davvero alto. «Che cosa ho sbagliato?» ti chiedevi dopo un incontro con lui, senza saperti dare risposta [...] Bassani te lo avrebbe detto ma non subito, non banalmente e in tempo perché 
tu ti potessi servire rapacemente dell'ammaestramento appena ricevuto, correndo ai ripari e correggendo il tuo sbaglio senza però avere imparato la lezione ${ }^{4}$.

C'è da chiedersi quanto, di quell'esperienza, sia stato drenato, tanti anni dopo, in queste osservazioni quasi aforistiche di Siciliano, contenute in una recensione a un'altra opera prima, Procida di Cordelli, di cui Siciliano fu pigmalione, osservazioni che sembrano muovere da un crocianesimo tollerante, avalutativo, evoluto, che (come vedremo più avanti) è il vero ponte fra la poetica bassaniana e quella di Siciliano:

All'editing, personalmente, non credo. I dattiloscritti o vanno restituiti tali quali sono, o vanno passati in tipografia, quando li si giudichi degni di stampa. Le loro opacità sono la loro vita. I figli nati non belli non possono venire rispediti al creatore perché gli dia una mano di bianco e li accomodi al nostro gusto: nella loro natura c'è già il segno di una definita e innegabile individualità. $E$ dall'imperfezione può nascere, in prospettiva, la salute, una vita diversa ${ }^{5}$.

Il magistero bassaniano era induttivo, dal puntillismo grammaticale («Bassani lesse, mi parve soddisfatto. Le sue mani si tenevano ferme al piano della scrivania, ma un indice si sollevava. Avevo usato un "sia... che". Con una certa stizza, Bassani mi disse che in italiano si scrive "sia... sia". Mi parve un'osservazione indolore. Avevo ventiquattro anni, da due insegnavo filosofia in un liceo: presi a stigmatizzare i miei studenti con una serie caparbia di "sia... sia"») 6 faceva risalire, nella coscienza dello scrittore consigliato e corretto, al massimo sistema: "Quei racconti che Bassani scartava, io li distruggevo - ciò avveniva non per compiacere lui, piuttosto perché mi accorgevo che quanto lui scartava era manchevole anzitutto dentro di me. Scrivere si tramutava in una ininterrotta scoperta di me stesso - un saldo che non si è mai chiuso da allora» ${ }^{7}$.

5 La conoscenza personale di Bassani, da parte di Siciliano, fu praticamente contemporanea alla scoperta di Bassani scrittore, che a distanza di anni Siciliano confessò su di lui influentissima: «Lessi quel racconto e me ne infatuai. Quella mia infatuazione, quella febbre tutta postadolescenziale che si può vivere quando si è intorno ai vent'anni e che fa callo per esuberanza col piacere della letteratura [...] insomma la lettura degli Occhiali d'oro cambiò il mio registro di valutazioni e interessi» ${ }^{8}$. Nei Racconti ambigui, è difficile non riconoscere l'ombra del Fadigati degli Occhiali d'oro - primo protagonista di queer novel della letteratura italiana - nell'omosessuale Holt ${ }^{9}$, intellettuale tedesco come lo Aschenbach della manniana Morte a Venezia ma anche ideale nipotino di Goethe e di Winckelmann sacralmente entusiasti di Roma e che muore in circostanze che anticipano in modo inquietante quelle della morte di Pasolini (tutte cose che finora non mi sembrano mai state notate dall'esigua critica addetta all'opera narrativa di Siciliano), di quel Pasolini che di Siciliano fu amico fin dagli anni di giovinezza di questi. E fu Pasolini, recensendo Autobiografia letteraria, la raccolta di saggi critici che conteneva il saggio su Bassani che sarà oggetto della più parte del nostro saggio, a legare maliziosamente Siciliano al Bassani divenuto vetrino d'analisi per la sua antica preda di talent scout, e ciò attraverso la figura di Fadigati e con un rovesciamento dialettico fra il vecchio e il giovane, tra realtà e finzione:

Ha scelto, mettiamo, l'impegno (la storia), ma sapendo benissimo che il limite di tale scelta è il pragma e l'ipocrisia. Il segno sotto cui avviene l'urto drammatico delle sue contraddizioni (sia pur accettate!) è la cattiva coscienza. Nella straordinaria requisitoria su Bassani [...] egli discute con se stesso: e si capisce abbastanza come Bassani sia rimasto offeso da queste pagine, che lo prendono, del resto tanto genialmente, a pretesto [...] Ammettiamo che Fadigati sia rinato sotto forma di un giovane moderno, operante negli anni Sessanta, in qualche modo 'diverso' (la sensibilità eccessiva del poeta, ecc.), che prenda a sua volta a pretesto 
di una sua correlazione dimostrativa Bassani, come Bassani aveva tanti anni prima a tale scopo preso lui. E che questo Fadigati critico analizzi se stesso, il proprio rapporto di colpevole connivenza coi padri miti, la propria incapacità di condannare il loro masochismo (la loro volontà a esser vittime del fascismo), la propria tendenza ad avere nostalgia della loro condizione - poetica perché profondamente precaria - di integrati ${ }^{10}$.

Curioso, a tal proposito, che in un'acuta recensione dei Racconti ambigui, Cesare Garboli già metaforizzasse sotto segno femmineo la prosa di Siciliano, leggendovi il controcanto in falsetto, la critica interna ai temi decadentistici declassati presso la piccola borghesia (ma segretamente, implicitamente cari all'autore...) nei racconti dell'amico ${ }^{11}$ Enzo:

[...] la prosa di Siciliano, piena di vezzi e ricca di isterie, ora frascheggia con gli aggettivi, ora civetta coi verbi, e sempre divaga, querula e rumorosa - non sta mai zitta la prosa di Siciliano - simile alle motorette negli ingorghi, sempre su di giri [...] si capisce anche l'ilarità di quella prosa, che smentisce il tema: mentre lo scrittore elargisce alla popolazione anonima dei suoi impiegati, delle sue professioniste, dei suoi squallidi poveri diavoli il pasto che era loro vietato, mentre li rende partecipi della 'vita', nello stesso tempo insinua un sospetto, fischietta un interrogativo, finge di non capire, divaga, e poi ammicca come chi la sa lunga, si fa beffardo: ma è chiaro, la sua ottica è decadente, ma è anche piccolo borghese, è anche 'italiana', quei grandi miti sono una balla, una balla da privilegiati, un'enorme turlupinatura [...] perché Siciliano ama quei privilegi, ama, anzi vuole, lui proprio, l'ambigua vita dello spirito, e teme di perderle, le sue belle e tenebrose tentazioni di decadente ${ }^{12}$.

7 E' vero che anche Giacomo Debenedetti, al dire di Siciliano, ravvisò fecondità di dissociazione interna nei Racconti ambigui: «L'originalità del libro, mi disse, consisteva nel fatto che lo scrittore vi si era posto in negativo nei confronti di se stesso, nei confronti del suo stile possibile. Crisi dell'io come crisi di un personaggio eventuale - o difficoltà nell'accettazione della psicologia» ${ }^{13}$. Ma più che mai notevole è che, quasi rendendo pariglia in termine d'elogio e quasi ricordando sotto traccia la recensione di Garboli ai suoi primi racconti, Siciliano individuasse nel rovesciamento dialettico la chiave del far critica letteraria dell'amico: «nessuno meglio di Garboli sa ragionare sui "partiti tecnici", anche se per vederli smossi, addirittura sfottuti, da qualcosa che sta più in profondo di essi e di cui essi sono epifania talvolta inconsapevole» ${ }^{14}$, «l'indagine di Garboli è governata da criteri dialettici o, meglio, dal criterio della negazione, che per Freud era il segno distintivo dell'atto del conoscere» ${ }^{15}$, e soprattutto

Quanto piace a Garboli scoprire l'istinto della persona in atto di sovvertire le maschere stilistiche, e dare espressione a qualcosa che elude la costruzione, il progetto! Affrontando in maniera spietata e diretta il problema dell'individualità, Garboli sguscia via dalle strettoie del romanticismo, proprio perché non chiede mai all'opera di dar voce al Volkgeist (un critico, finalmente!, per niente platonizzante) ${ }^{16}$.

8 Tornando alla quota femminile, o di Anima junghiana, attribuita sia da Pasolini sia da Garboli all'intelligenza creatrice e critica di Siciliano, non meno curioso è che, vent'anni dopo, nel forte delle polemiche susseguite alle rivelazioni ${ }^{17}$, da parte di Siciliano, circa le prevaricazioni e i complotti della 'corte' neoavanguardista di Giangiacomo Feltrinelli che portarono nel 1963 alla cacciata di Bassani dalla casa editrice $^{18}$, per due volte Alberto Arbasino, al pari di Pasolini omosessuale, in difetto di argomenti alludesse volgarmente a Siciliano con epiteto di genere ${ }^{19}$. Contro il Gruppo '63, che in Arbasino aveva esponente di spicco, Siciliano nel 1965 aveva del resto avventato un libro, Prima della poesia, vigorosamente panflettistico già dal titolo 
(la neoavanguardia come eterno, irrisolto prolegomeno all'opera, un «prima» progettuale che non finalizza mai, un'impalcatura davanti al nulla: «fra progetto e opera, ho sempre scelto l'opera», opporrà Siciliano in un'intervista del 1992) ${ }^{20}$ : è il momento di massima sintonia con la poetica e con le posizioni critiche di Bassani, quali manifestate in una celebre, fremente intervista ${ }^{21}$ del ferrarese di quegli anni, dopo la clamorosa affaire con Feltrinelli, finita peraltro nelle aule dei tribunali e cui abbiamo già accennato, con tanto di accuse a Bassani di elegismo consolatorio, «Liala '60" e altre perfidie strumentali e strategicamente screditanti. Un libro, Prima della poesia, che in molti luoghi appare acutamente parafrastico delle ironiche demistificazioni bassaniane verso chi aveva, contro lo stesso Bassani, esercitato una forma di terrorismo intellettuale: il libro di un intelligente seguace, magari più munito o più aggiornato ideologicamente del suo mentore. Se infatti la polemica contro la meccanica linguistica dei neovanguardisti («Lo spirito della confezione, pure tutto esteriore, soddisfa l'esigenza di una società che va perdendo il senso della persona. Esprimersi non è più individuare un nesso di emozioni, ma semplicemente far parata di varie possibilità combinatorie (attitudini da cucina, da sartoria)») ${ }^{22}$ ripete la refrattarietà di Bassani verso quella che a questi sembrava una mimesi passiva e consenziente, despiritualizzata e di scuola francese (l'école du regard) ${ }^{23}$, della pseudoggettività alienante del secondo capitalismo (per cui, marxianamente, la società prende la forma di un rapporto tra cose: la barbarie della ragione secondo Adorno), è notevole e personale in Siciliano l'analisi del carattere regressivo e adialettico della 'spinta in avanti' della neoavanguardia:

A me sembra, tanta smaniosità a percepire il futuro prima ancora di averlo vissuto, un bisogno patologico: - il bisogno di chi capovolge nell'opposto la spinta regressiva da cui si sente afflitto. Spingersi velleitariamente verso il domani, rifiutando l'oggi e la sua contraddittoria incidenza di futuro e passato, equivale a dichiarare la propria impotenza a sentirsi nel profondo liberi e affrancati da qualsiasi condizionamento [...] Ma tale smania di modernità è piuttosto diventata una superstizione che non consente al mutare reale delle cose di essere appunto in movimento: ma le congela, finge di questo una eternità, che seppure è progrediente, è bloccata nel suo divenire come l'universo immaginato da Parmenide [...] l'uomo che si autodefinisce "moderno" e "aggiornato", "teso verso il futuro", non tollererà mai di sostituire le proprie idee con altre diverse: di far dichiarare fallimento alla propria "modernità", di dirla "superata", "non attuale", deliziosamente antiquata. Di sentirsi cioè immerso nella storia ${ }^{24}$.

Il silenzio della persona poetica, ed etica, è l'unica speranza che si apre di fronte al piccolo borghese di oggi per difendere le posizioni che riesce a conquistare. Il suo fine è l'immobilità storica, o lo schizomorfismo: nella visione dissociata è la sua forma: nel dialettizzare la vita presente si accorge che il suo volto di classe si annullerebbe ${ }^{25}$.

L'adorazione dell'esistente, camuffata in prospezione di modernità, conduce alla disumanizzazione dell'arte: «Se esserci espressione è esserci persona, per cui un valore e un orizzonte di fini, il piccolo borghese nega tutto ciò con ribrezzo» ${ }^{26}$, stante che «la poesia è evento individuo, irripetibile, relazione singolarissima fra soggettività e oggettività, il cui accadere non è elevabile a legge, ma è legge in sé, vitalità che non conosce ripetizione $\aleph^{27}$, come Siciliano sentenzia con tautologia crociana (incrociata con la proposizione aristotelica della poesia essere vivente al pari di qualsiasi altro) anch'essa di sapore bassaniano. Arbasino, col suo "pettegolezzo orchestrato in elefantiasi ${ }^{28}$, secondo Siciliano pecca innanzitutto contro l'individualità sensibile e creatrice, tradisce e svia l'autobiografismo che mette in programma: 
La partenza, l'approccio all'argomento, è in Arbasino sempre autobiografico: mai però che l'autobiografismo lo infanghi, mai che i suoi peccatucci e inclinazioni traspaiano dichiaratamente, mai che vengano a galla personali indulgenze. Come, allora, saltare in groppa all'autobiografia, al proprio sguardo, che perciò stesso è personale, fingendo che non lo sia? Arbasino ha coltivato l'illusione di poter scantonare al dilemma trascrivendo gerghi, con l'effetto, sempre più soffocante, di non riuscire a scapolare al proprio gergo, alla musica del proprio cinismo livellatore ${ }^{29}$.

Arbasino rimarrà sempre, per Siciliano, l'emblema, e la testa di turco, di una concezione del romanzo antiumanistica, gratuita come un gioco di pazienza: «Vennero i francesi, gli strutturalisti, "chi più ne ha più ne metta", e la narrazione diventò per lo scrittore de L'anonimo lombardo una sorta di mobyle del quale gli interessavano piuttosto le giunture che le volute, più il sistema di equilibrio che il colore che l'adorna» ${ }^{30}$. Il movimento da cui è trascinato Arbasino rinnega di fatto l'impeto delle avanguardie rivoluzionarie primonovecentesche con un conservatorismo mandarinesco, col formalismo proprio dei classicismi più reazionari: «L'avanguardia storica fu, nel suo complesso, un atto di vitalità generale ricco di un contenuto esplosivo [...] La neoavanguardia volle codificare quella vitalità, farne un galateo, un insieme di regole di comportamento: volle rovesciare un guanto» ${ }^{31}$. Secondo Siciliano, il cifrario gelido, statico, astrattamente nombriliste della neoavanguardia ripete i vizi dell'ermetismo anteguerra: «Il neoermetismo si sposò alla spregiudicatezza neoindustriale. L'artigianato del romanziere venne considerato attività obsoleta, casomai femminile, $\mathrm{o}$ casomai da contestare con l'antiromanzo, il libro che, pensato contro il lettore, rivendicava la purezza di un'autonomia espressiva del tutto celibe, del tutto autistica ${ }^{32}$. Si noti come Siciliano, di contro alla brutalità sterile che ascriveva all'avanguardismo 'neoermetico', si ritagliasse vittimisticamente la parte femminile che Pasolini e Garboli gli avevano riservato quale risorsa dialettica sia come romanziere sia come critico: tanti anni dopo, in controcanto amebeo e durante la baruffa giornalistica susseguente alla rivelazioni di Siciliano su quello che Garboli definì il «teppismo padronale $»^{33}$ di Feltrinelli, Arbasino si divertirà a rinnovare il ludo, appuntando a Siciliano che è letteratura "che non ha le palle» ${ }^{34}$ quella che non regge la polemica e si lamenta dei colpi bassi nella lotta per le idee...

L'anno seguente l'uscita di Prima della poesia, nel 1966, Siciliano pubblica su «Nuovi Argomenti ${ }^{35}$ L'anima contro la storia, un lungo e pregnante saggio su Bassani che rielabora idee già espresse molto sommariamente in un articolo apparso su «Mondo operaio» nel $1964^{36} \mathrm{e}$ che rifluirà in Autobiografia letteraria del 1970 ${ }^{37}$. È un saggio dove le armi della demistificazione ideologica sono volte adesso verso il suo grande editor di pochi anni prima, pur nel rispetto dovuto a una personalità consentanea (con l'ambiguità del Doppelgänger di cui parlava Pasolini, nella citazione da noi sopra recata). Il titolo del saggio sottolinea la dicotomia che, sulla scia delle rapsodiche ma geniali analisi dedicate da Pasolini a Bassani, secondo Siciliano informa l'opera bassaniana: l'anima contro la storia, intimismo che invano aspira a una storicizzazione dell'esperienza, anzi che adibisce uno storicismo giustificazionista per proteggere il godimento solitario e inconfessabile dell'«anima», bloccata nella beata regressione agli anni che pur erano stati della sventura della polis, gli anni del Fascismo. La situazione bassaniana è per Siciliano già sottesa alla prova poetica di esordio (Storie dei poveri amanti) e a quelle dell'immediato dopoguerra, sotto l'egida di quell'ermetismo ${ }^{38} \mathrm{che}$ fu bersaglio inquieto della saggistica di Siciliano negli anni '60 (di passaggio, già lo 
abbiamo riscontrato in Prima della poesia) tanto che nello stesso 1966 il poligrafo romano poteva scrivere:

Il concetto di "assenza", tanto caro ai giovani poeti e critici del tempo, non aveva alcun contenuto positivo: indicava soltanto l'incapacità a dialettizzare vita e letteratura, a scendere criticamente nella fibra dell'inquietudine, a liquidare patemi d'ascendenza cattolica, i quali impedivano la definizione materiale della situazione storica. L'ermetismo servì solo a rimuovere reali necessità di conoscenza, sempre in virtù di un ideale estetizzante e letterario di vita ${ }^{39}$.

Di Te lucis ante Siciliano dice: «Sono settenari di colta sostenutezza, compatti, quasi impenetrabili in un loro (basta una lettura a voce alta) sonante languore. Questa languidezza è una via per capire la natura della crisi (e la reazione ad essa), patita da Bassani $»^{40}$. Il dramma dell'olocausto rinvierebbe in Bassani all'elezione individuale («Gli è rimasto il rimorso d'essere scampato al genocidio, il turbamento di chi non sa se, con questo, sia stato toccato da un carisma») $)^{41}$, ancora una volta contro la storia, e la condizione di vittime del genocidio sarebbe scambiata con quella di una dignità borghese esautorata e rimpianta: Bassani «non pronuncia la parola decisiva. Vittime persuase certamente! Ma non perché ebrei: - perché borghesi, come tutti i borghesi italiani. Il giudizio non si invera: si rinchiude dentro la tradizione letteraria. La sua supposizione immanentistica non diviene parola. Il suo grido non si fa immagine: il poeta teme che possa suonare offesa alla memoria dei padri» ${ }^{42}$. Lì e qui l'influenza delle pagine pasolinane su Bassani sembra inconfutabile: «Lo storicismo diventa la chiave per capire gli avi, e contemporaneamente non offenderli. Così, la lotta col padre (archetipica nella piscologia ebraica) si svolge in Bassani non su un piano di parità, ma di accettata dipendenza. Ed è questo difetto (ancora il vizio puberale dell'indulgenza verso se stessi!) che inficia, e inficierà in lui il bisogno di risolvere dialetticamente il passato ${ }^{43}$. Confrontiamo col lucido affondo di Pasolini:

[...] la ristrettezza numerica e mentale delle borghesia ebrea di Ferrara e la grandiosità che le viene conferita dalla "diaspora" e dalla tragedia della persecuzione. Non so quale dei due momenti prevalga. Forse nessuno dei due. Se ciò che su tutto prevale è in fondo il rimpianto del piccolo-borghese ebreo di non essere un piccolo-borghese qualsiasi, e il suo sforzo terribile per sembrare tale. In realtà tutta la poesia di Bassani trova la sua fonte in questo rimpianto. Non essere un borghese qualsiasi ${ }^{44}$ !

Per Pasolini (secondo il quale è questo, di Bassani, «il sedimento, in tanto limpida e pronta intelligenza, di una mascherata immaturità e inermità, e insieme d'un po' di diabolico atteggiamento dell'astuzia, recuperante nel razionale ciò che nell'impeto irrazionale qualche volta si arena» $)^{45}$ il dispositivo storicizzante nella narrativa di Bassani non ha il fine di inverare il passato alla luce del presente ma di distanziarlo, preservandolo in quanto passato, impreziosendolo in quanto destinato a passare nello stesso momento in cui è fatto presente alla rievocazione ${ }^{46}$ :

[...] in Bassani il "tempo" del corrente modo di narrare - il tempo apparentemente logico, che giustappone in prospettive del resto arbitrarie i fatti ad esso incorporati - è sostituito da un tempo distaccato da quei fatti, che quindi si dispongono a sé, liberi dalla sua logica lineare e finalistica. Essendo la vicenda "conclusa", è uscita dal tempo, è divenuta un cristallo, con le sue misure interne assolute, e la sua superficie che può mostrare indifferentemente ora l'una ora l'altra delle sue facce ${ }^{47}$.

Gli avvenimenti di cui parla Bassani sono tutti visti non solo come passati, ma come destinati a passare [...] e l'evocazione avviene attraverso una forte presenza psicologica del narratore [...] forte almeno quanto è debole il valore del presente, del momento in cui il narratore parla: che corrisponde al momento in cui il 
narratore parla: che corrisponde al momento in cui gli avvenimenti narrati erano destinati a non essere più. Insomma l'integrazione figurale di Bassani è la fine di tutto, un'accorata illusorietà della storia: le sue figure in questo trovano compimento, e quindi prevale in esse un senso meglio patetico che politico della vita. Il mondo di Bassani risulta quindi, nella sua apparente interezza e lucidità politica e storicistica, profondamente diviso e sfuggente: la consolazione è il "barlume di allegrezza" di chi sente accoratamente la dolcezza nella disperazione, l'eterno nel fuggitivo. La chiarezza storiografica o la passione azionistica ne sono attutite e come vanificate ${ }^{48}$. trascendentale della pietas in Bassani si oblitera, perché a lui manca, dopo il decadentismo, l'appoggio di un sistema di valori obiettivi che la consenta. La sua prima persona è problematica per necessità. A queste condizioni, la pietas diventa un modo di rimozione») $)^{50}$ verso i padri, i quali garantivano l'immobilità mitica del paradiso infantile, per Pasolini decideva anche del partito stilistico (Citati scrisse poi: «La sua [di Bassani] mancanza di grazia è quasi inconcepibile in un vero scrittore. Allinea la sua rete di avverbi, le sue frasi convenzionali, e sembra compiacersi che la lingua sia un sepolcro di metafore decadute, di immagini spente, di cose mancate») ${ }^{51}$ della narrativa bassaniana:

Essi [Cassola e Bassani] mescolano allo stile sublimis, fondamentale alla loro ispirazione elegiaca e civile, una lingua parlata come lingua dei padri (naturalmente borghesi) che, visti nella luce della memoria si nobilitano, diventano oggetto di recherche: e con essi si nobilita la loro lingua parlata, quell'italiano medio, che dopo averli respinti da sé - per una violenta protesta storica e ideologica, mettiamo l'antifascismo - li richiama col fascino di un luogo promesso e perduto, una normalità poetica in quanto struggentemente ontologica ${ }^{52}$.

Il loro [di Cassola e di Bassani] stile [...] non è che una serie continua e sia pur coperta di "citazioni" del linguaggio borghese e piccolo-borghese usato dai padri e dai nonni professionisti e dalle loro cerchie provinciali ${ }^{53}$.

Tra Pasolini a Siciliano circola o coincide una visione dell'opera bassaniana di intimismo all'ombra della letteratura, a voler giocare con la famosa formula di Benn su Thomas Mann, ovvero di specchiamento di un primum inevoluto protetto dalla letteratura. «Bassani si trovò respinto sempre più verso le secche di un mancato storicismo, di défaillance dialettica tra vita e letteratura, restando per lui la letteratura un primum indiscusso» ${ }^{54}$, scrive Siciliano nel suo saggio, inferendo tara di classe nel giudizio 'letterario' di Bassani sul fascismo, in termini non dissimili da quelli che userà per Croce nel grande saggio del $1971^{55}$ sul filosofo: «Il fascismo fu per lui una malattia dello spirito, non una conseguenza delle idealità borghesi. I giovani intellettuali, che si formarono alla sua scuola in quegli anni, si sono trovati, nella maggioranza dei casi, nella impossibilità di far coincidere i propri sentimenti di rivolta con la conoscenza razionale del mondo in cui si trovavano ${ }^{56}$. Un freno ulteriore in Bassani, secondo Siciliano, all'analisi storicizzante e un'implicita letterarizzazione della sua situazione, 
autobiograficamente trasposta, dinanzi ai marosi del fascismo e della guerra, viene dalla condizione ebraica:

[Bassani] Subì il pogrom: non ne vide i caratteri classisti (che riguardavano tutti gli uomini liberi, e non i soli ebrei), che un punto di vista materialista poteva illuminare. Bassani si fermò alla propria anima colpita. L'ottimo studente di liceo rivendicò la bontà degli studi regolari compiuti: egli fa parte della comunità nazionale in spirito, dicano quel che vogliono $\mathrm{i}$ fascisti. Alla continuità storica, "imperiale" e "ariana", oppone la continuità risorgimentale d'osservanza crociana. Comunque, è quella ferita a spiegare il suo sforzo storicistico: ma è la cristallizzazione sulla propria anima colpita (indulgenza e reticenze incluse), che denuncia la spiritualizzazione del medesimo $0^{57}$.

L'interpretazione del finale di Una lapide in via Mazzini, da parte di Siciliano, insiste sulla destoricizzazione mitica ebraizzante: «Geo riprende le spoglie dei padri erranti, "a capo chino". Anche questa è metafora emblematica: è nostalgia per i miti ancestrali» ${ }^{58}$. Dopo Siciliano, solo Ada Neiger ha voluto trovare segni e metafore regressive, ebraicamente caratterizzate, nelle storie bassaniane («Il ritorno al ghetto, come soluzione salvifica del problema giudaico, è una vena, ora sotterranea e impercettibile, ora affiorante e che percorre tutta la narrativa a sfondo ebraico di Bassani. Già nei titoli delle opere bassaniane, ricorrono espressioni che richiamano gli spazi delimitati, la separazione, l'isolamento») ${ }^{59}$, ma molto prima Fortini aveva puntato il dito sulla natura difensiva della Sehnsucht bassaniana quale ritorno ideale al ghetto:

[...] non ha fatto della propria esistenza la sede di una operazione di disinganno e di conversione, come l'eroe di Proust; non c'è nessuno smascheramento né ulteriore ricupero dei suoi modesti Guermantes; c'è un desiderio di immutabilità che finisce col credere che valore e immutabilità coincidano [...] la «magna domus» non è solo il materno ghetto, il talèd sotto cui rifugiarsi dalla tempesta imminente; è la difesa dalla storia e anche dalla propria storia, interiore, la difesa della "pigra brace che è tanto spesso il cuore dei giovani" 60 .

Da parte marxista, in termini che oggidì suonerebbero senz'altro politicamente scorretti, Gaetano Trombatore aveva deprecato, non senza ironia, l'acronica geremiade «dell'ebreo», incapace di riconoscere le proprietà storiche e l'impegno a esse dovuto:

[...] la situazione particolare dell'ebreo, il quale è anch'egli uncinato dall'ingranaggio di quei fatti, ma, anche se ne rimane vittima, ne è preso, per così dire, tangenzialmente, giacché per lui quelle angosciose vicende smarriscono la loro puntualità concreta e impegnativa, e si inseriscono invece, come un anonimo anello, in una catena di sevizie e di persecuzioni la cui storia si perde nel passato e nella memoria degli uomini; e pertanto quel nodo di fatti, oltre e forse più che appartenente alla storia italiana, si presenta come un episodio, affine a tanti altri episodi, di una parallela e maligna e segreta storia tutta intessuta di inevitabili e fatali calamità. Da questa singolare disposizione nasce un modo di essere, che nell'atto stesso di aderire ai fatti, tende a guardarli con disimpegno e con distacco, come da una biblica extratemporalità ${ }^{61}$.

E l'anno seguente, il 1960, Bertacchini e Cusatelli, due compagni di cordata di Siciliano in «Palatina», la rivista parmense nella quale egli era stato introdotto da Bertolucci ${ }^{62}$, insistono sul tema: Bertacchini ripeteva quasi, di Trombatore, l'osservazione che «Le Storie di Bassani possono rendere l'impressione di una serie di cronache organizzate ab aeterno, staticamente ferme, immobili sotto la luce di una biblica e incorrotta extratemporalità» ${ }^{63}$, mentre Cusatelli asseriva che «il sentimento religioso che rappresenta l'autentico nucleo storico dell'ebraismo, non più costretto al rigore 
teologico della tradizione mosaica, torna accettabile allo spirito moderno dello scrittore [= Bassani] come dolcezza di costumanze familiari» ${ }^{64}$. che la critica recente ${ }^{69}$ ha definito come centrale e struttivo nell'opera bassanish viene da Siciliano (che prima ancora di Garboli lo affrontò da pioniere) ricondotto alla topica letteraria («Il ritorno al cimitero, come al luogo dell'illuminazione e della pacificazione, al luogo che rimette in moto gli istinti vitali fiaccati, è obbligato nella narrativa di Bassani da Clelia Trotti ai Finzi-Contini). Ma, piuttosto che di una bruciante esigenza del cuore - mai che l'animo del narratore ne sia insidiato, quando invece ne è confortato -, è un ritorno che rimanda al tropo letterario, particolarmente ne Gli occhiali d'oro» $)^{70}$, sì che, raccordandosi ai «Sepolcri, con le suggestioni vichiane e le prefigurazioni storicistiche che contiene», «Bassani sfiora volentieri l'intenzionalità concettuale di tanta narrativa moderna, ma poi la ripara dietro l'allusione culturale, la riconduce a proporzioni prevedibili» ${ }^{71}$. Con ciò, secondo Siciliano, Bassani mutua dall'esterno l'kidea foscoliano-carducciana dell'impegno civile», fa propria l'«esaltazione del "tono alto e virile" del poeta-vate»" e pertanto «è per i rapporti con l'Ottocento italiano che si definisce il limite di Bassani. La linea "civile" in lui non si ritaglia modernamente, quando pure ne avrebbe l'ambizione; e accade che egli resti un delicato elegiaco, coatto dentro la letteratura che più ama: - mentre il lettore, inviato a spaziare nell'universo della storia e degli imperativi morali, si ritrova dentro un museo» ${ }^{73}$. Un «delicato elegiaco»... Dei due corni della definizione pasoliniana, quale in precedenza abbiamo citato («elegiaco e civile»), Siciliano taglia via, come avventizio, il secondo, e il primo, solitario sebbene alto nella classifica di merito, viene a sovrapporsi, di fatto, con le rimostranze del Gruppo '63 contro Bassani...

In conformità a queste risultanze esegetiche, è dell'inesorabile, inventariale puntualità dei tanti oggetti designati col nome commerciale nelle Cinque storie ferraresi e poi nei 
Finzi-Contini, degli oggetti cognominati come persone, dei famosi marchionimi ${ }^{74}$ bassaniani, che per primi Siciliano e Brian Moloney nello stesso anno, il 1966, segnalarono la funzione di «rinchiudere il ricordo nel particolare, stampigliarlo dentro la precisione: impedirgli di fuggire. Un puntiglio di pittore di natura morta, tradotto in sintassi da incisi, da quegli arricchimenti interni della frase che i linguisti chiamano "espansione" $\rangle^{75}$ : così Siciliano, mentre Moloney contemporaneamente scriveva che Bassani «ha bisogno di ammassare fatti, luoghi, nomi, date, figure, per poter contrastare con questo cumulo di certezze "quel poco che il cuore ha saputo ricordare" $\gg^{76}$. Una fortezza del ricordo, invero antiproustiana, perché ordinato magazzino che sventa l'entropia angosciosa col superstizioso amuleto della fredda nominazione mercantile degli oggetti del passato e che il registro linguistico grigiamente notarile difende, con dissimulata sacralizzazione borghese (come già voleva Pasolini), dall'aggressione delle ideologie, dai risentimenti edipici, dai ridimensionamenti affettivi, dalle rese dei conti con la storia di tutti. Questa ossessione apotropaica, secondo Siciliano (e Moloney intendeva parimenti), investe anche la forma dramatis, il minuzioso catasto dei luoghi, dei microeventi pseudofondamentali, callidi succedanei di un'articolazione diegetica che non articola, che non sviluppa, che si blocca e vuole bloccarsi su una vie antérieure di assolutezza intangibile: «La richiesta di questi fondali si precisava, nel corso della narrazione, come un soccorso strategico. Tanta puntigliosità, sembrava necessaria a calamitare gli elementi drammatici, a fonderli, quasi non riuscissero a diventare autonomi centri di forza» ${ }^{77}$.

Eppure, o proprio perciò, in Bassani «ogni racconto sottintende un mistero, un interrogativo che lo sviluppo della vicenda deve chiarire» ${ }^{78}$ e «lo scioglimento, o accertamento del caso, è il più delle volte la formulazione di un problema, la sua proposta in termini di chiarezza: niente altro ${ }^{79}$, scrive Siciliano delle Storie ferraresi. La perfetta, protettiva chiusura sul passato attinge l'ingiudicabilità dello stesso, la trionfante inespugnabilità del senso di esso. Nell'Airone, nota Siciliano in un'appendice del 1968 al saggio di «Nuovi Argomenti» e che verrà pubblicata soltanto in volume, «il "passato remoto" acquista una saldezza sconosciuta, sale a tonalità dominante. Il petrarchismo parrebbe dissolversi, e al suo posto subentrare la coscienza del narrare in prosa: affidare i segni della propria vita a simboli fallibili, accettare che l'irrisolto si spieghi con se stesso, rischiando ogni offuscante contraddittorietà» ${ }^{80}$. È qui che il giudizio di valore del sinuoso e corrosivo saggio di Siciliano passa a segno euforico, è qui che la poetica della bêtise 'ispirata', dalla densità insopportabile e inconoscibile allo stesso creatore, è qui che la poetica dell'intransigenza avalutativa di ascolto del creatore rispetto ai suoi personaggi stringe in alleanza il critico-narratore e il narratore oggetto del suo studio. Eanche in questo caso si deve rilevare che il succitato, precisamente coevo, stupendo e davvero sottovalutato saggio di Moloney giungeva a conclusioni parallele («il narratore viene così rivelato come colui che non comprende» ${ }^{81}$; «personaggio centrale che è un enigma per se stesso, che è incapace, o meglio, non vuole esaminare più da vicino il travaglio del suo cuore e della sua mente. Questo personaggio è il narratore ${ }^{82}$; «i motivi che provocano le azioni, anche quelle del narratore, rimangono un enigma ${ }^{83}$ ) e che una recensione di Piovene ai Finzi-Contini, oggi tranquillamente dimenticata, opponendo Bassani a Robbe-Grillet («offerte al nulla senza un minimo di dolore») e cioè assecondando la polemica bassaniana di quegli anni contro la neoavanguardia cugina dell'école du regard, celebrava di Bassani «personaggi misteriosi come tutti gli esseri vivi, perché fatti di molti strati, perché li complica il bisogno di interpretarli, una realtà piena di ombre, non ottica né descrittiva» ${ }^{84}$ (tanti 
anni dopo, Maria Bellonci, ricordando il Premio Strega assegnato a Bassani nel 1956 per le Cinque storie ferraresi: «un luogo del mondo chiamato Ferrara: dove è necessario che accadano storie indimenticabili di gente che Bassani ci mostra nelle palpitazione estrema del loro esistere: visioni poetiche fermate un istante prima di scomparire, e raggianti in quel momento nell'energia dei loro segreti») ${ }^{85}$. Proprio sul nucleo ermetico all'interpretazione dei personaggi bassaniani si erano appuntate le riserve della critica marxista, ad esempio di un Ferretti, nel 1959, prima ancora della pubblicazione dei Finzi Contini, contro la bassaniana «rinuncia a capire la vita, a penetrarla, e quindi la tendenza a ritrarsene spaventati, a evaderne insomma» ${ }^{86}$, ciò che si deposita, narrativamente, nell'«individuo solo, incomprensibile, isolato in una realtà non modificabile, in un mondo ostile e inesorabilmente uguale e a se stesso» ${ }^{87}$, ed è qui che si consuma la separazione di giudizio, pur su comuni parametri storicistici, fra Siciliano e la linea, per così dire, Fortini-Trombatore-Ferretti ${ }^{88}$. Così si esprimeva Ferretti, ancora, sul tema del personaggio-istrice, portatore di irrazionalità refrattaria, tautologica, autogiustificantesi:

In un mondo dove la vita scorre immobile e sempre eguale, dove gli uomini celano in fondo a se stessi un nucleo segreto e impenetrabile, dove in definitiva l'intimo significato di ogni vicenda è da cercare nell'enigma di ciascun uomo, il solo modo di capire la realtà, di cogliere il senso segreto che essa nasconde, è quello della 'folgorazione' poetica, dell'intuizione improvvisa e irrazionale ${ }^{89}$.

Ma era la discendenza crociana («teologia negativa, nei confronti della poesia» ${ }^{90}$, scrisse Siciliano nel suo notevole saggio su Croce) che riavvicinava potentemente Siciliano a Bassani sul fronte del quid ineffabile, che per essi due faceva equazione tra individualità incommensurabile dell'opera artistica, natura apofatica della sintesi intuizioneespressione, autotelismo impenetrabile del personaggio narrativo. Nel Siciliano critico l'inconoscibile imperiosità dell'invenzione, la «scandalosa necessità che è della poesia» ("hélas!, scriveva Bataille, "come si può perdere tempo su libri alla cui creazione l'autore non sia stato manifestamente costretto?" $\rangle^{91}$, «il compimento di un'opera di poesia è qualcosa che scatta misteriosamente a mezzo dei processi inventivi, e soggioga chi scrive defilandolo senza rimedio dal quadro [...] Quando l'opera slitta via dal suo autore, entrambi si salvano ${ }^{92}$ ) si coniuga all'avvincente opacità dell'opera per lo stesso suo autore («io credo che uno scrittore sia ciò che a lui stesso è sconosciuto»" ${ }^{93}$, «non lo dico per vezzo, ma conosco pochissimo questo romanzo» ${ }^{94}$, detto della Principessa $e$ l'antiquario, che Siciliano pubblicò nel 1980) e al barthesiano piacere del testo per il lettore («Dell'eros il rapporto che abbiamo con un testo possiede le abulie, le repentine e indomabili rapinosità, la quiete intensa e profonda e - seguendo ancora Barthes l'indicibilità») ${ }^{95}$. Non sarà stato per caso, quindi, che la notizia che Bassani era stato colpito dall'Alzheimer trovò in Siciliano un commento ben intonato a questa persuasione centrale nel fare letterario sia di Bassani sia di Siciliano medesimo: «La necrosi del tessuto della memoria è uno degli attentati più misteriosi e devastanti che la natura può compiere al nostro spirito poiché, pur nell'apparente obliterazione, noi restiamo chi siamo, un io irriducibile che non riesce a sottrarsi alla luce accecante del proprio destino» ${ }^{96}$. Si rileggano adesso le proposizioni più famose e altre meno citate di Bassani intorno all'inesprimibilità di ciò che è espresso, alla concretezza dell'ineffabile ${ }^{97}$, al personalismo irriducibile dell'opera d'arte insomma:

Si dice in genere che la mia narrativa è di derivazione ottocentesca: non lo credo affatto, proprio per il motivo che da parte mia non sono mai state tentate costruzioni o ricostruzioni di tipo psicologico [...] Il fatto che io abbia sentito così a 
fondo l'idealismo comporta la certezza, per me, che l'io profondo è ineffabile.

È effabile soltanto ciò che si dice, che si fa ${ }^{98}$. altrettanto soprendente affinità con le posizioni di Lukács ${ }^{99}$, Bassani aveva sempre elevato a barriera rispetto alla letterarietà avulsa e seriproducente del decadentismo $\mathrm{e}$ alla disgregazione formalistica della neoavanguardia! Bassani rivendica per sé oltranza anche rispetto ai grandi eversori dell'io narrativo tradizionale, nel romanzo primonovecentesco: «[...] io non ho la fede dei miei immediati predecessori (Proust, Joyce) che l'io profondo sia effabile, conoscibile; io non ci credo, non ci credo più» ${ }^{100}$. È un autentico dogma antipsicologistico, quello di Bassani («C'è andato a letto, Micól, a letto con Malnate? Io mi ritraggo e dico di non saperlo perché effettivamente non lo so. Voglio mantenermi veritiero e non voglio indagare, perché sono soltanto un romanziere») ${ }^{101}$, una confessa mistica del personaggio:

Quasi nessuno scrittore ha rispetto per i propri personaggi, li considera come delle persone realmente vissute o realmente viventi, quasi nessuno scrittore pretende di mettersi in una posizione morale nei confronti della propria opera [...] Questo è il punto fondamentale. Introdurre il lettore in una dimensione letteraria, in un rapporto letterario di carattere morale e religioso ${ }^{102}$.

La figlia di Bassani, Paola, in una recente intervista ha fornito al proposito una testimonianza personale importante e un rilievo interpretativo inedito ed euristico:

[...] nella sua [= di Bassani] opera le morti sono sempre "incerte", sempre "fuori campo", mai vero sigillo ma attraversamento in cui si può continuare a vivere: una morte dietro le quinte, senza la scena tragica e conclusiva. Il rapporto con la morte come momento di passaggio non c'è, insomma, perché il personaggio è ineffabile così come lo è il suo trapasso. Del resto mio padre pronunciava spesso la parola "ineffabile" ${ }^{103}$.

Ma, nel saggio di Siciliano su Bassani del 1966, dopo questa grande convergenza scatta l'affondo contro l'opera, i Finzi-Contini, che pur aveva acceso la miccia della polemica sanguinosa con quella neovanguardia l'anno prima attaccata, da posizioni per tanti versi bassaniane, in Prima della poesia:

È un'opera nata da un profondo riflusso di emozioni, è il segno del venir meno della tensione morale. Così lo stile si ricompone, si paluda in movenze architettoniche complesse: si inchioda a formule evocative, infarcite di elementi ritardanti. Sfugge all'articolazione degli Occhiali d'oro ${ }^{104}$.

Cosa differenzia colui che ha ricevuto la ferita che sappiamo, e il giovane che chiede amore a Micól? Niente. La ferita è lì: piuttosto che osservarla, "conoscerla”, egli rientra in grembo al passato: chiede il visto per il giardino, cerca un ricovero, una protezione, una difesa ${ }^{105}$.

Siciliano ripete insomma ai Finzi-Contini l'accusa di accartocciamento consolatorio, che Garboli contesterà anni dopo (in occasione della querelle fra Bassani e De Sica per l'adattamento cinematografico del romanzo) rivendicando all'opera tonicità prospettica, e non depressa coltura, del tempo perduto, «recupero doloroso della vita da quell'archeologia, da quel gusto di cose morte (le opaline di Micòl) verifica critica della propria vitalità, anche se mista alla pietà per la loro fine, quella pietà che è il rimorso dei vincitori ${ }^{106}$. Siciliano invece fulminava giudizio di classe, rinnovando l'acido critico da Pasolini versato in precedenza: «Col Giardino sale a galla il rimpianto reale per il mondo dei Finzi-Contini. La funzione antifascista svanisce. Bassani è ferito perché la guerra ha travolto il bene del passato - che sono Micòl e il professor Ermanno. Che è, insomma, il piccolo paradiso dei borghesi, dove i profumi saranno 
mefitici, ma non per questo meno paradisiaci» ${ }^{107}$. Ebbene, in un'intervista giornalistica del 1978 allo stesso Siciliano, mai raccolta in volume e del resto mai citata dalla critica, è notevole che Bassani, parlando della perdita di speranza del presente a paragone con le generose illusioni resistenziali e dell'impossibilità di un esilio 'formativo' dall'Italia, si lasciasse andare a un rimpianto di accento talleyrandiano, in cui il vigore della denuncia faceva antitesi con la voluttà rimozionale, così dando ragione a Siciliano e a Garboli al contempo: «Non c'è da prendere nemmeno la via dell'esilio. Chi va in esilio si propone di tornare: qui non si può tornare. Eppure questo è un Paese dove stiamo anche molto bene. Oh, quanto sconteremo la douceur de vivre che ci siamo concessi!» ${ }^{108}$. Siciliano, imperniandosi sulla formula lapidaria che dà il titolo al suo saggio, anima contro storia, e guardando indietro dai Finzi-Contini giù fino agli esordi poetici di Bassani, finalmente nega una dinamica evolutiva alla carriera bassaniana, rimasta secondo lui interna a un bozzolo aureo e cresciuta per addizioni sur place:

L'anima contro la storia. Lo storicismo in Bassani è una prospettiva che egli non riesce ad attuare. La sensibilità del borghese è prevaricatoria di ogni possibilità conoscitiva. Da qui nasce la sua esitazione espressiva, e il suo calamitarsi a moduli ottocenteschi. Insomma, i primi impulsi, affatto letterari, allo scrivere il narcisismo dei suoi versi giovanili, non sono stati superati: egli ha tentato la via più difficile per aggiunzione, attraverso un potenziamento sostanziale ${ }^{109}$.

Ma nell'appendice del'68 sull'Airone si festeggia la svolta, l'omicidio-suicidio (quest'ultimo, del protagonista Edgardo Limentani) del passato che la scrittura bassaniana aveva imbalsamato per lunghi anni e attraverso tante opere, imbalsamato come l'airone dietro la vetrina illuminata dell'impagliatore che persuade Edgardo, quasi per una rivelazione, all'atto 'rivoluzionario', anti-wertheriano perché di comprensione-accettazione e non di protesta: Edgardo «si uccide, dopo una giornata trascorsa a caccia, fuggendo richiami domestici, i richiami di un passato che gli si scopre incapsulato dentro l'inafferrabile compattezza degli oggetti. Il suo sguardo corre sulle cose e le congela - travolto da una sorda ebbrezza» ${ }^{110}$. Secondo Siciliano, con Edgardo è Bassani stesso che si libera della sua vecchia pelle per consumazione intera, $\mathrm{e}$ terapeutica perché eutanatica, dell'esperienza 'morta', reificata che era stata propria della vita di Edgardo e della poiesi dello scrittore stesso:

[...] il suicidio è qui operazione di trasferimento lampante: è il modo di accettare, attraverso interposta persona, il destino di certe scelte espressive. Di accettarle, però, agendole: di lasciarsi condurre avanti dal daimon fino a sorprenderlo in servitù. L'intelligenza di uno scrittore si misura sempre in questo: nel saper rovesciare a proprio vantaggio gli svantaggi della sua natura ${ }^{111}$.

31 Anche in questo caso è da notare come Siciliano si muova criticamente in una sorta di dialogo a distanza con Garboli (l'altro influencer è, come abbiamo visto, Pasolini), stavolta in anticipo su di lui (il saggio di Garboli sull'Airone è dell'anno seguente, del '69): per l'Airone Garboli parla di «vita finalmente immune da se stessa [...] contrita di esistere [...]. Per oggetti morti, scrittura morta [...] la copia, l'imitazione, il calco di una lingua morta» ${ }^{112}$. Se attraverso la vicenda di una giornata di Edgardo Limentani «un fiaccato sentimento della vita si trasforma in felicità: ma è la decadente, estetica felicità da annientamento»" ${ }^{133}$, è che "Bassani pensa che non si possa "mortificare" oggetti già morti. Flaubert ruota su se stesso: si ribella ai suoi argomenti morti, li contesta e cerca di riportarli in vita» ${ }^{114}$. Come anche aveva inteso Siciliano, Bassani ha, secondo Garboli, bevuto fino in fondo della conoscenza di sé l'amaro calice, ha rovesciato per saturazione le sue ossessioni fuoriuscendo a nuova vita. 
Se adesso tiriamo le fila dell'attenzione critica di Siciliano verso il suo ammirato ma ingombrante editor degli esordi, dovremo parlare di un esito chiaroscurale di giudizio, se anche, come ebbe a dire Pasolini (il quale, come citavo in precedenza, ci informa del malumore nella ricezione del saggio del ' 66 da parte di Bassani), requisitoria non fosse. Per Siciliano «il male ha il volto dell'estetismo, il bene pretende una decisione, una rottura, una scelta» ma «quella [del milieu di Bassani] borghesia era sedotta da se stessa, dalla fine qualità dei propri comportamenti» ${ }^{115}$ e Bassani, fino all' Airone, usa lo strumento storicizzante per ibernare e preservare il passato, non per metterlo in movimento verso il presente, per coglierne la dinamica. L'alta coscienza letteraria, l'alto artigianato letterario fanno asepsi che inibisce i germi socialmente venefici di quel passato e permette libera accoglienza delle forme più disparate e stimolanti, ma, come in un ospedale, rende tanto più presente la morte nell'isolamento:

Il torto di Bassani [...] fu quello di concepire la letteratura come una "città ideale" - dico proprio la tavola dipinta conservata al Palazzo Ducale di Urbino, quella piazza vuota di uomini, concepita come una scena perfettamente prospettica, le cui uniche tracce di vita sono alcuni gerani ai balconi. Una natura morta. Ma non si può neanche muovere rimprovero per questo. Quell'algida concezione della letteratura ebbe in sé una inimitabile capacità dinamica - ospitare le più diverse esperienze senza coercizioni. Lo storicismo puro ha come propria divinità tutelare la libertà, anzitutto la libertà d'espressione, una volta che essa si dispieghi per necessità, senza trucchi di sorta e per trasparente autonomia dei fini. Ma quella purezza ha in sé anche qualcosa di morboso e di funebre ${ }^{116}$.

In un'intervista del 1981, ricchissima di spunti di poetica, Siciliano tracciava un'idea di letteratura come avventura, come salto nel buio, che, se fatta retroagire sul gran saggio su Bassani, non avrebbe potuto che riservare una sede limbale all'opera bassaniana:

La letteratura-letteratura, a lungo andare, è noiosa, nel senso che non completa l'ovvia parabola narcisistica che l'esprimersi comporta. Chi fa letteraturaletteratura, per dirla con un'espressione gergale, si ferma alla prima osteria. Ma scrivere è un'avventura, una lacerazione, un sentiero che porta progressivamente verso un orizzonte in cui, di noi stessi, sappiamo sempre di meno ${ }^{117}$.

È tuttavia quel limbo, fra arte pura e impegno, che Bassani accettava come suo, non per anemica insufficienza, sibbene quale risultante di tensioni opposte, quale sconfitta romanticamente eroica:

L'arte deve essere pura o deve raggiungere la purezza per una specie di fallimento dell'impegno. Io non credo che si possa rimproverare alla mia letteratura di essere pura, perché la poesia è sempre pura, se è realizzata. Io adesso, pensando a tutto quello che ho fatto, posso compiacermi - nonostante il mio impegno, che vi garantisco è vero e serio - di avere fallito e di esser rimasto solo un artista, un artista puro $^{118}$.

\section{NOTE}

1. Cronologia, a cura di R. Manica, in E. Siciliano, Opere scelte, a cura e con un saggio introduttivo di R. Manica con la collaborazione di S. Casini, Milano, Mondadori, 2011 (pp. XI-CXLI), p. LXXXVI.

2. Ivi, pp. LXXXIX-XC. 
3. Cfr. A. Debenedetti, Bassani amava il romanzo, in E. Siciliano, Bassani, a cura di A. Debenedetti, Roma, Elliot, 2016 (pp. 7-11), pp. 10-11.

4. Ivi, p. 9.

5. Cfr. E. Siciliano, Il tesoro nell'isola (1973), in Id., La bohème del mare. Dieci anni di letteratura 1972-1982, Milano, Mondadori, 1983 (pp. 132-134), pp. 132-133.

6. Cfr. E. Siciliano, Gli anni di «Racconti ambigui» (1993), in Id., Opere scelte, cit. (pp. 1148-1167), p. 1151.

7. Ivi, pp. 1152-1153. Un ricordo scherzoso, ma la lezione salò il sangue dell'apprendista: «So benissimo quanto ossessivamente cerco di evitare, scrivendo, ripetizioni (e spesso non mi riesce), o quanto, altrettanto ossessivamente, lotto con la punteggiatura» (cfr. E. Chierici, Enzo Siciliano, La Nuova Italia, 1981, p. 5).

8. Cfr. E. Siciliano, Giorgio Bassani editore, in M. I. Gaeta (a cura di), Giorgio Bassani. Uno scrittore da ritrovare, Roma, Fahrenheit 451, 2004 (pp. 29-44), p. 39.

9. Cfr. E. Siciliano, Friederich Holt e il suo ventitré giugno, in Id., Racconti ambigui, Milano, Garzanti, 1972 (1963), pp. 141-173.

10. Cfr. P. P. Pasolini, Il discorso critico svolto come fiaba (1970), in Id., Saggi sulla letteratura e sull'arte, vol. II, a cura di W. Siti e S. De Laude con un saggio di C. Segre, Milano, Mondadori, 2004 (1999) (pp. 2507-2510), pp. 2508-2509. Una lettura recente incentrata sulle contraddizioni del rapporto di Bassani narratore con l'omosessualità è V.L. Puccetti, Bassani e l'omosessualità, «Cuadernos de Filología Italiana», vol. 23, 2016, pp. 27-41.

11. Cfr. E. Siciliano, Gli anni di «Racconti ambigui», cit., p. 1153.

12. Cfr. C. Garboli, Il male non c’è (1963), in Id., La stanza separata, Milano, Scheiwiller, 2008 (1969) (pp. 121-127), pp. 123-124 e 126.

13. Cfr. E. Siciliano, Gli anni di «Racconti ambigui» (1993), cit., p. 1155.

14. Cfr. E. Siciliano, Stanza separata (1969), in Id., Autobiografia letteraria, Milano, Garzanti, 1970 (pp. 385-389), p. 386.

15. Ivi, p. 385.

16. Ivi, p. 387.

17. Cfr. E. Siciliano, I miei autori, in Id., Romanzo e destini, Roma, Theoria, 1992 (pp. 9-23), pp. 9-19.

18. Sul caso clamoroso e sui suoi retroscena cfr. almeno G. C. Ferretti, Un editore di gusto, in Giorgio Bassani critico, redattore, editore, a cura di M. Tortora, Roma, Edizioni di Storia e Letteratura, 2012 (pp. 165-176), pp.174-175 (in nota a p.174, bibliografia sulle voci più recenti di ricostruzione della vicenda) e P. Italia, All'insegna di un «vero maestro». Bassani e «Paragone», ivi (pp. 143-162), pp. 155-158 (a p.162 bibliografia utile, anche se non completa, sulla polemica divampata sui giornali dell'epoca).

19. Cfr. A. Arbasino, Una letteratura spiegata da uscieri e pizzaioli, «Tuttolibri», $\mathrm{n}^{\circ} 788$ («Andiamo, signore mie: siamo seri, non facciamo le pazze!»), febbraio 1992, e Id., Caro Enzo è solo livore, «Repubblica», 26-27 gennaio 1992 («Però che meschinità, signora mia, ritirar fuori ancora queste vecchie sulle poetiche di trent'anni fa»). Occorre comunque dire che le rivelazioni di Siciliano provocarono reazioni scomposte e anzi incresciose nei vecchi appartenenti al Gruppo'63, reazioni peraltro nemmeno indirettamente implementanti sul piano culturale. Ad esempio, su «Tuttolibri» del gennaio 1992, $\mathrm{n}^{\circ}$ 786, Arbasino, nell'articolo Abbiamo pulito il tinello, esibiva stentoreo le medaglie del Gruppo («ci sono parecchi docenti universitari di successo, due ex deputati al Parlamento, un direttore di Raitre»), con coerenza rivoluzionaria invero... Su «Tuttolibri» del febbraio 1992, $\mathrm{n}^{\circ} 788$, Renato Barilli scriveva una lettera al giornale per lamentare, lui invece, l'esclusione dalle onorificenze (In quel gruppo c'ero anch'io: perché nessuno l'ha ricordato?, «Sono costernato nel constatare che l'articolista ha eliminato totalmente ogni riferimento alla mia persona»). Sulla «Repubblica» del 26-27 gennaio 1992, Caro Enzo è solo livore, Edoardo Sanguineti era lapidario: «[...] dal punto di vista letterario e culturale Siciliano è un poveruomo». 
20. Cfr. E. Siciliano, Le avanguardie del'63 gelarono il romanzo (intervista di Nico Orengo), «Tuttolibri», n 788, febbraio 1992.

21. G. Bassani, In risposta (III) (1963), in Id., Opere, a cura e con un saggio di R. Cotroneo, Milano, Mondadori, 2004 (1998), pp. 1215-1219. Sugli interventi militanti di Bassani contro quella che egli riteneva la deriva neoavanguardistica nella letteratura degli anni del boom economico, cfr. in particolar modo A. Roncaccia, Emergenze polemiche del discorso critico bassaniano negli anni sessanta e settanta, in D. Capodarca e T. Matarrese (a cura di), Indagini sulla narrativa di Giorgio Bassani a dieci anni dalla sua scomparsa, Atti del Convegno di Studi di Ferrara (14 ottobre 2010), Firenze, Le Lettere, 2012, pp. 117-135, passim.

22. Cfr. E. Siciliano, Prima della poesia, Firenze, Vallecchi, 1965, p. 15.

23. Cfr. ad esempio G. Bassani, In risposta (I) (1959), in Id., Opere, cit. (pp. 1169-1173), p. 1171: «L'impassibilità mortuaria del Voyeur e della Jalousie evoca direttamente la dittatura del grande capitale industriale, il "moderno" qualunquismo neocapitalista e neopositivista».

24. Cfr. E. Siciliano, Prima della poesia, cit., pp. 15-16.

25. Ivi, p. 82.

26. Ivi, p. 83.

27. Ivi, p. 97.

28. Ivi, p. 111.

29. Ivi, p. 109

30. Cfr. E. Siciliano, Le brame di Arbasino (1974), in Id., La bohème del mare. Dieci anni di letteratura 1972-1982, cit. (pp. 206-210), p. 207.

31. Cfr. E. Chierici, Enzo Siciliano, cit., p. 5.

32. Cfr. E. Siciliano, I miei autori, cit., p. 12.

33. Cfr. Bassani: resto nel mio giardino (intervista di M. Serri), «Tuttolibri», $n^{\circ} 786$, gennaio 1992.

34. Cfr. A. Arbasino, Abbiamo pulito il tinello, ivi.

35. 1 n.s., febbraio-marzo, pp. 80-108.

36. Cfr. E. Siciliano, Bassani a tu per tu, «Mondo operaio», vol. XVII, n² 2-3, 1964, pp. 57-60.

37. Cfr. E. Siciliano, Autobiografia letteraria, cit., pp. 98-135.

38. "Io sono nato dunque fiorentino», ammise Bassani nella fondamentale Intervista inedita a Giorgio Bassani. Istituto Italiano di Cultura di New York in cooperazione con la radio Italiana, 1966, in R. Antognini e R. Diaconescu Blumenfeld (a cura di), Poscritto a Giorgio Bassani. Saggi in memoria nel decimo anniversario della morte, Milano, LED, 2011 (pp. 611-623), p. 614.

39. Cfr. E. Siciliano, I poeti sull'Aventino (1966), in Id., Autobiografia letteraria, cit. (pp. 325-329), p. 328.

40. Citiamo da E. Siciliano, Bassani, cit., che comprende la quasi totalità degli scritti di Siciliano su Bassani (p. 18).

41. Ivi, p. 18.

42. Ivi, p. 20.

43. Ivi, pp. 24-25.

44. Cfr. P. P. Pasolini, Giorgio Bassani, «Il romanzo di Ferrara». I: «Dentro le mura» (1974), in Id., Saggi sulla letteratura e sull'arte, vol. II, cit. (pp. 1990-1994), p. 1994.

45. P. P. Pasolini, Bassani (1953), in Id., Saggi sulla letteratura e sull'arte, vol. I, cit. (pp. 499-506), p. 500 .

46. Indaga l'intento di decostruzione critica nei confronti di Bassani da parte di Pasolini sceneggiatore della Lunga notte del '43, V. L. Puccetti, Io nascosto, redistribuzione di materiali bassaniani e Pasolini antagonista nella «Lunga notte del '43» di Florestano Vancini, «Sinestesie», vol. 14, 2016, pp. 187-207.

47. Cfr. P. P. Pasolini, Referto per «Botteghe oscure» (1951), in Id., Saggi sulla letteratura e sull'arte, vol. I, cit. (pp. 389-393), p. 391.

48. Cfr. P. P. Pasolini, La confusione degli stili (1957), ivi (pp. 1070-1088), p. 1084. 
49. Cfr. E. Siciliano, Bassani, cit., p. 26.

50. Ivi, p. 27.

51. Cfr. P. Citati, Bassani cerca il cuore nascosto dei Finzi-Contini, «Il Giorno», 21 febbraio 1962.

52. Cfr. P. P. Pasolini, Nuove questioni linguistiche (1964), in Id., Saggi sulla letteratura e sull'arte, vol. I, cit. (pp. 1245-1270), p. 1249.

53. Ivi, p. 1255.

54. Cfr. E. Siciliano, Bassani, cit., p. 32.

55. Cfr. E. Siciliano, La proiezione fuori della figura (Benedetto Croce) (1971), in Id., La voce di Otello, Milano, Mondadori, 1982 (pp. 171-200), p. 175: «[...] un'antica aristocrazia agraria, già degradata a spuria borghesia, pure con i suoi fondachi al sole, - spuria perché non pervenuta a costituire società, a fissare i propri istituti linguistici, e tale diventata per inerzia. È una classe che ha subìto l'eredità dei principi ideali dell'umanesimo, senza tradurli in problema, che li ha ridotti a buon senso contadino, li ha mescolati alla tradizione cattolica, a riti pagani più arcaici; che non ha accolto nel suo insieme le crisi rigenerative delle borghesie europee dell'Ottocento: - non ha tradotto alcuna propria crisi in un qualche sistema razionale: non ha subito il fascino della scienza».

56. Cfr. E. Siciliano, Bassani, cit., p. 32.

57. Ibid.

58. Ivi, p. 34.

59. Cfr. A. Neiger, Bassani e il mondo ebraico, Napoli, Loffredo, 1983, p. 29.

60. Cfr. F. Fortini, Di Bassani [1963, per la parte citata], in Id., Saggi italiani, vol. I, Milano, Garzanti, 1987 (pp. 251-260), p. 257.

61. Cfr. G. Trombatore, Materia e stile, in Id., Scrittori del nostro tempo, Palermo, Manfredi, 1959 (pp. 107-111), p. 108-109.

62. Cfr. Cronologia, cit., p. LXXXIX.

63. Cfr. R. Bertacchini, Appunti sul semitismo di Bassani, in Id., Figure e problemi di narrativa contemporanea, Rocca San Casciano, Cappelli, 1960 (pp. 301-338), p. 336.

64. Cfr. G. Cusatelli, Caratteri dell'opera narrativa di Bassani, «Palatina», vol. V, $\mathrm{n}^{\circ} 20,1960$ (pp. 9-19), p. 11.

65. Cfr. E. Siciliano, Bassani, cit., p. 22.

66. Ivi, p. 33.

67. Cfr. Cronologia, cit., pp. LXXXII-LXXXv.

68. Cfr. E. Siciliano, Bassani, cit., p. 39.

69. Cfr. soprattutto P. Pieri, Memoria e giustizia. Le "Cinque storie ferraresi» di Giorgio Bassani, Pisa, ETS, 2008, passim ma particolarmente pp. 195-220; ma vedi anche M. Actis-Grosso, Poetica sepolcrale e topologia bassaniana nel «Giardino dei Finzi-Contini», in A. Perli (a cura di), Giorgio Bassani: la poesia del romanzo, il romanzo del poeta, Ravenna, Giorgio Pozzi, 2011, pp. 119-140, e F. Secchieri, La 'meditatio mortis' nella scrittura di Bassani, in Indagini sulla narrativa di Giorgio Bassani a dieci anni dalla sua scomparsa, cit., pp. 161-177.

70. Cfr. E. Siciliano, Bassani, cit., p. 35.

71. Ivi, p. 36.

72. Ivi, p. 36.

73. Ivi, p. 37.

74. Cfr. B. Moloney, Tematica e tecnica nei romanzi di Giorgio Bassani, «Convivium», vol. XXXIV, $\mathrm{n}^{\circ} 5$, 1966, pp. 484-495.

75. Cfr. E. Siciliano, Bassani, cit., p. 21. Per la tecnica dell'inciso in organismo sintattico fortemente subordinante e per il registro referenziale-burocratico (di cui Pasolini, come citato in precedenza, aveva fornito interpretazione ideologica) in Bassani, gli studi linguistici di riferimento sono: H. Haller, Da «Le storie ferraresi» al «Romanzo di Ferrara»: varianti nell'opera di Bassani, «Canadian Journal of Italian Studies», vol. 1, n 1, 1977, pp. 74-96; I. Baldelli, Verso una 
lingua comune: Bassani e Cassola, in Id., Varianti di prosatori contemporanei (Palazzeschi, Cecchi, Bassani, Cassola, Testori), Firenze, Le Monnier, 1965, pp. 46-75; Id., La riscrittura 'totale' di un'opera: da «Le storie ferraresi» a «Dentro le mura» di Bassani, in Id., Conti, glosse e riscritture dal secolo XI al secolo XX, Napoli, Morano, 1988, pp. 241-265; T. Matarrese, Bassani e la lingua del romanzo, in A. Chiappini e G. Venturi (a cura di), Bassani e Ferrara: le intermittenze del cuore, Ferrara, Corbo, 1995, pp. 47-63; E. Testa, Sulla lingua delle «Cinque storie ferraresi», in P. Pieri e V. Mascaretti (a cura di), Cinque storie ferraresi. Omaggio a Bassani, Pisa, ETS, 2008, pp. 55-64; Id., «Dire tutto»: lessico e sintassi dell'«Airone», in Giorgio Bassani: la poesia del romanzo, il romanzo del poeta, cit., pp. 171-183.

76. B. Moloney, Tematica e tecnica nei romanzi di Giorgio Bassani, «Convivium», cit., p. 488.

77. Cfr. E. Siciliano, Bassani, cit., p. 37.

78. Ivi, p. 28.

79. Ivi, p. 29.

80. Ivi, p. 57.

81. B. Moloney, Tematica e tecnica nei romanzi di Giorgio Bassani, cit., p. 493.

82. Ivi, p. 491.

83. Ivi, p. 489.

84. Cfr. G. Piovene, Restituiscono Marx agli intellettuali, «L'Espresso», 4 marzo 1962.

85. Cfr. M. Bellonci, Io e il Premio Strega, Milano, Mondadori, 1987, p. 47.

86. Cfr. G. C. Ferretti, Bassani e Cassola tra idillio e storia (1959), in Id., Letteratura e ideologia: Bassani, Cassola, Pasolini, Roma, Editori Riuniti, 1974 (pp. 17-161: ma su Bassani fino a p. 65), p. 45.

87. Ivi, p. 46.

88. Che fu anticipata dall'articolo, pressoché dimenticato oggi ma assai intelligente su solco gramsciano intrattabilmente ortodosso, M. T. Lanza De Laurentiis, Bassani, la storia e l'enigma, «Società», vol. XII, nº 5, 1956, pp. 1007-1012.

89. Cfr. G. C. Ferretti, Bassani e Cassola tra idillio e storia, cit., p. 36.

90. Cfr. E. Siciliano, La proiezione fuori della figura (Benedetto Croce), cit., p. 182.

91. Cfr. E. Siciliano, Qualche distinguo (1966), in Id., Autobiografia letteraria, cit. (pp. 351-355), p. 355.

92. Cfr. E. Siciliano, Due storie (1969), ivi (pp. 86-97), p. 89.

93. Cfr. E. Chierici, Enzo Siciliano, cit., p. 7.

94. Ivi, p. 9.

95. Cfr. E. Siciliano, Forfait del testo (1975), in Id., La bohème del mare. Dieci anni di letteratura 1972-1982, cit. (pp. 217-219), p. 218.

96. Cfr. E. Siciliano, La memoria offesa di Giorgio Bassani, «La Repubblica», 1 maggio 1996.

97. Su ciò mi permetto di rinviare a V.L. Puccetti, L'«aprosdóketon» nel racconto bassaniano, in Poscritto a Giorgio Bassani. Saggi in memoria del decimo anniversario della morte, cit. p. 143-162, e a V. L. Puccetti, Bassani e Hawthorne, in Giorgio Bassani: la poesia del romanzo, il romanzo del poeta, cit. (pp. 33-56), pp. 46-47. Cfr. anche C. Terrile, «È effabile soltanto ciò che si dice, che si fa». La scrittura dell'immanenza nell'ultimo Bassani, «LaRivista», n 3, 2015 (pp. 61-79), pp. 62-65.

98. Cfr. F. Camon, Il mestiere di scrittore: conversazioni critiche, Milano, Garzanti, 1973, p. 58.

99. Su ciò cfr. il fitto e impegnato intervento di A. Perli, Bassani critico e la poetica della realtà, in Giorgio Bassani critico, redattore, editore, cit. (pp. 13-33), passim ma soprattutto pp. 15, 21 e 25, e anche F. Bausi, Contributi alla critica di se stesso. Giorgio Bassani e la letteratura italiana, ivi, pp. 35-61, passim.

100. Cfr. A. Dolfi, Meritare il tempo (1981), in Id., Giorgio Bassani, Una scrittura della malinconia, Roma, Bulzoni, 2003, p. 171.

101. Cfr. F. Camon, Il mestiere di scrittore: conversazioni critiche, cit., p. 65.

102. Cfr. Intervista inedita a Giorgio Bassani. Istituto Italiano di Cultura di New York in cooperazione con la radio Italiana, 1966, cit., p. 616. 
103. Cfr. S. Amrani e M. P. De Paulis-Dalembert (a cura di), Le forme dell'impegno di Giorgio Bassani. Intervista a Paola Bassani, in Id., Bassani nel suo secolo, Ravenna, Giorgio Pozzi, 2017 (pp. 395-406), p. 406.

104. Cfr. E. Siciliano, Bassani, cit., p. 40.

105. Ivi, p. 41.

106. Cfr. C. Garboli, L'occhio del cinema dà soltanto quello che vede (1971), in Id., La gioia della partita, Milano, Adelphi, 2016 (pp. 208-211), p. 270.

107. Cfr. E. Siciliano, Bassani, cit., p. 39.

108. Cfr. E. Siciliano, I rimpianti di Bassani, «La Stampa», 24 ottobre 1974.

109. Cfr. E. Siciliano, Bassani, cit., p. 45.

110. Ivi, p. 46.

111. Ibid.

112. Cfr. C. Garboli, L'airone (1968), in Id., La stanza separata, cit. (pp. 343-349), p. 344.

113. Cfr. E. Siciliano, Bassani, cit., p. 59.

114. Cfr. C. Garboli, L'«Airone» di Giorgio Bassani (1971), in Id., La gioia della partita, cit. (pp. 119-120), p. 120.

115. Cfr. E. Siciliano, La memoria offesa di Giorgio Bassani, cit.

116. Cfr. E. Siciliano, I miei autori, cit., p. 14.

117. Cfr. E. Chierici, Enzo Siciliano, cit., p. 5.

118. Cfr. Intervista inedita a Giorgio Bassani. Istituto Italiano di Cultura di New York in cooperazione con la radio Italiana, 1966, cit., p. 621.

\section{RIASSUNTI}

L'articolo affronta nella sua prima parte il rapporto editoriale e culturale fra Giorgio Bassani e Enzo Siciliano, con particolare riguardo alla comune polemica contro la neoavanguardia. Nella seconda e più vasta parte, si analizza la lettura critica che Siciliano fece dell'opera narrativa dell'autore ferarrese in un ampio e importante saggio del 1966 (con un'appendice del 1968), in cui Siciliano, sulla scia di alcune osservazioni di Pier Paolo Pasolini, intendeva demistificare ideologicamente l'approccio storicistico di Bassani, mostrando i tratti conservativi borghesi della sua retrospezione memoriale. Il punto d'incontro, su un piano di poetica, fra Siciliano e Bassani risiede comunque nella concezione del personaggio di romanzo, enigma per lo stesso autore, al quale si impone con una forza e con un'evidenza la cui natura rimane ineffabile e irriducibile.

The paper addresses in its first part the editorial and cultural relations between Giorgio Bassani and Enzo Siciliano, with particular regard to their common polemics against the Italian New Vanguard (Neoavanguardia). In its second and longer part, it deals with the critical reading that Siciliano made of Bassani's narrative work in a 1966 large and important essay, to which he added an appendix in 1968. In the wake of Pasolini's perceptive remarks, Siciliano endeavored to demystify Bassani's historical approach, by stressing the bourgeois-conservative overtones of his attitude towards past and memory. One should however stress that Siciliano and Bassani share the same conception of the character, which they describe as an enigma even to the author, and which forces itself upon the reader with relentless force. 
Dans sa première partie l'article aborde le rapport éditorial et culturel entre Giorgio Bassani et Enzo Siciliano, en particulier leur commune polémique avec la «neoavanguardia». Dans la seconde partie, plus longue, on analyse la lecture critique qu'a menée Siciliano de l'œuvre narrative de l'auteur ferrarais dans un ample et important essai de 1966 (avec un appendice de 1968). Dans le sillage de quelques observations faites par Pier Paolo Pasolini, Siciliano dénonçait dans cette étude la mystification idéologique de l'approche historique de Bassani, montrant les aspects bourgeois conservateurs de son mémorialisme rétrospectif. Le point de rencontre, en termes de poétique, entre Siciliano et Bassani, réside néanmoins dans la conception du personnage de roman, énigme pour son auteur même, à qui il s'impose avec une force et une évidence dont la nature demeure ineffable et irréductible.

\section{INDICE}

Parole chiave : Giorgio Bassani, Cesare Garboli, Pier Paolo Pasolini, Enzo Siciliano, Benedetto Croce, ebraismo, neoavanguardia, omosessualità, retorica del personaggio letterario, storicismo Keywords : Giorgio Bassani, Cesare Garboli, Pier Paolo Pasolini, Enzo Siciliano, Benedetto Croce, historicism, judaism, neoavanguardia, homosexuality, rhetorics of the literary character

Mots-clés : Giorgio Bassani, Cesare Garboli, Pier Paolo Pasolini, Enzo Siciliano, Benedetto Croce, historicisme, judaïsme, neoavanguardia, homosexualité, rhétorique du personnage littéraire

\section{AUTORE}

\section{VALTER LEONARDO PUCCETTI}

Università del Salento 